\title{
O ESTUDO DE PRECESSÃO DA ÓRBITA DE MERCÚRIO NO ENSINO MÉDIO
}

\author{
R. F. F. DA CUNHA* \\ Colégio Pedro II, Campus Realengo II, Rio de Janeiro, RJ, Brasil. \\ A. C. TORT $^{\dagger}$ \\ Instituto de Física, Universidade Federal do Rio de Janeiro, RJ, Brasil.
}

\begin{abstract}
Resumo
A lei da gravitação universal de Newton é a base da nossa compreensão da dinâmica do Sistema Solar e sob certas condições, de uma boa parte do universo observável. No nível do ensino médio, as órbitas dos planetas são apresentadas como sendo elípticas, com o Sol ocupando um dos focos. Isto é verdadeiro se considerarmos apenas a interação gravitacional entre o Sol e o planeta. Mas um o planeta não sofre apenas a força gravitacional exercida pelo Sol. Outros planetas também exercem forças gravitacionais e, além disso, outros efeitos devem ser levados em conta como por exemplo a oblaticidade do Sol ou os efeitos previstos pela versão relativística da gravitação newtoniana, a teoria geral da relatividade. O resultado final é que as órbitas planetárias não são fechadas, isto é: as órbitas precessam. É possível introduzir este tema no ensino médio? Os autores acreditam que a resposta é afirmativa e que o fenômeno da precesssão das órbitas dos planetas pode ser estudado com o auxílio de softwares modernos muitos deles gratuitos e da fácil aprendizagem. Neste trabalho, discutiremos o fenômeno da precessão em particular, o efeito relativístico na precessão de Mercúrio e sugerimos sua implementação em sala de aula por meio do software educacional Modellus.
\end{abstract}

Palavras-chave: A física e seu ensino, gravitação newtoniana, precessão de órbitas planetárias; precessão de Mercúrio; tecnologias no ensino de física.

*Aluno de doutorado do Programa de Pós Graduação em Ciência, Tecnologia e Educação do Centro Federal de Educação Tecnológica Celso Suckow da Fonseca, Unidade Maracanã, RJ, Brasil. E-mail: ricardofagundescp2@ gmail.com

$\dagger$ E-mail: tort@if.ufrj.br 


\begin{abstract}
Newton's law of universal gravitation underpins our understanding of the dynamics of the Solar System and under certain conditions, of a good portion of the observable universe. In high school, the orbits of the planets are announced as ellipticals, with the Sun occupying one of the foci. This is true if we consider the gravitational interaction between Sun and planet only. But a planet does not suffer the gravitational force exerted by the Sun only, but also by the gravitational forces exerced by others planets. Besides that other effects must be considered such as the oblaticity of the Sun and the effects predicited by relativist version of Newton's law of universal gravitational. The final result is that the planetary orbits are not closed, that is: the orbits precess. Is it possible to introduce this topic in high school? The authors believe that the answer is affirmative and the phenomenon of precession of orbits can be studied using modern softwares, many of them free and easy to learn. In this paper we will discuss the phenomenon of precession and, in particular, the relativistic effect on the precession of Mercury and we suggest its implementation through the Modellus educational software.
\end{abstract}

keywords: physics education, newtonian gravitation, precession of orbits, precession of Mercury, technology in physics education.

\title{
1 Introdução
}

Individualmente, os planetas não sofrem apenas a ação da força gravitacional exercida pelo Sol. Pequenas perturbações fazem com que o semieixo da órbita originalmente elíptica gire lentamente. A velocidade com que o semieixo gira é a velocidade de precessão da órbita. Estas pertubações têm várias origens, por exemplo, o fato de o Sol não ser perfeitamente esférico, a interação com os outros planetas, ou ainda os efeitos da teoria geral da relatividade, i.e., o fato de o Sol deformar o tecido de espaço - tempo [1].

Entre todos estes efeitos perturbativos, a correção da relatividade geral aplicada a um planeta particular, Mercúrio, é talvez a que mais chama a atenção.

Por que Mercúrio? A razão é simples: sua proximidade do Sol faz com que ele sofra com maior intensidade os efeitos da deformação da geometria do que os outros planetas, não que o efeito seja grande, mas comparativamente é o mais fácil de ser observado. De um ponto de vista histórico, a precessão de Mercúrio é a pedra no sapato da gravitação newtoniana que tanto sucesso tivera na explanação da mecânica do Sistema Solar. As primeiras medidas da precessão da órbita de Mercúrio não concordavam com a predição newtoniana, a discrepância entre o valor teórico e o valor observado era de aproximadamente 43 segundos de arco por século [2]. Inicialmente, a discrepância pareceu confirmar a existência de um novo planeta entre o Sol e Mercúrio, Vulcano, mas com o tempo, esta hipótese foi descartada e os 43 segundos de arco por século transformaram-se num dos três testes clássicos da então recém proposta teoria geral da relatividade (TGR) ou mais propriamente: teoria relativística da gravitação. 
No presente trabalho propomos a introdução deste problema nas discussões sobre a gravitação no nível do ensino médio. A complexidade téorica do problema não impede que ele possa ser discutido de forma semi-quantitativa combinando uma descrição do fenômeno da precessão apsidal com sua concretização por meio de um software de fácil aprendizagem, o Modellus como o leitor poderá constatar na próximas seções.

\section{2 Órbitas em campos de forças centrais}

Quando consideramos o sistema planeta - Sol, isoladamente, isto é, o problema não-perturbado, o período radial $T_{\text {radial }}$, o intervalo de tempo necessário para que o planeta descreva uma oscilação radial completa e o período orbital, o intervalo de tempo necessário para que a partícula descreva uma volta completa em relação a um ponto de referência sobre a órbita são iguais, ou seja, a razão entre os períodos $T_{\text {radial }} / T_{\text {orbital }}$ é igual à unidade. Isto é verdadeiro para a lei da gravitação newtoniana; caso a interação gravitacional obedecesse à lei de Hooke, a razão entre os períodos $T_{\text {radial }} / T_{\text {orbital }}$ seria metade da unidade, conforme será argumentado a seguir. O leitor pode verificar examinando a Figura 1 que se escolhermos o perihélio (o ponto em que a Terra está mais próxima do Sol) como ponto de referência, nosso planeta descreve um elipse completa durante a qual a distância radial vai do valor mínimo ao valor máximo (o afélio) e volta ao valor mínimo em um intervalo de tempo que coincide com uma oscilação radial completa. $\mathrm{Na}$ Figura 2, a lei de força corresponde a um oscilador harmônico isotrópico. Neste caso, a cada duas oscilações radiais completas teríamos uma elipse completa.

As afirmações acima decorrem do teorema de Bertrand que nos garante que as únicas forças centrais que levam a órbitas limitadas e fechadas são a lei do inverso do quadrado $f(r) \propto r^{-2}$, caso da gravitação newtoniana, e a linear $f(r) \propto r$, caso do oscilador harmônico isotrópico. Para outras leis de força centrais, é possível encontrar órbitas fechadas, por exemplo, órbitas circulares, mas isto dependerá de uma escolha particular das condições iniciais.

Outro modo de enunciar o teorema de Bertrand é afirmar que os dois únicos potenciais que levam a órbitas fechadas para todos os estados ligados são o potencial de Kepler: $U(r) \propto-r^{-1}$, e o potencial do oscilador harmônico isotrópico: $U(r) \propto r^{2}$. No caso do potencial de Kepler, os estados ligados são definidos pela condição $E<0$, onde $E$ é a energia mecânica total; já no caso do potencial do oscilador harmônico isotrópico $E>0$.

O teorema de Bertrand é tratado extensivamente na literatura. Demonstrações padrão podem ser estudadas em [3, 4]. Demonstrações alternativas podem ser estudadas, por exemplo, em [5]; uma tradução do original francês para o português pode ser consultada em [6].

\section{Precessão}

Há vários tipos de precessão, por exemplo, na mecânica do sólido, precessão significa uma mudança na direção do eixo de rotação do corpo. É o que acontece com o pião e o giroscópio. Na astronomia, o termo precessão refere-se em geral à precessão axial (no caso da Terra, precessão dos equinócios) como ilustrado na Figura 3, e à precessão apsidal, ou no caso de um planeta que orbita o Sol, precessão do periélio, Figura 

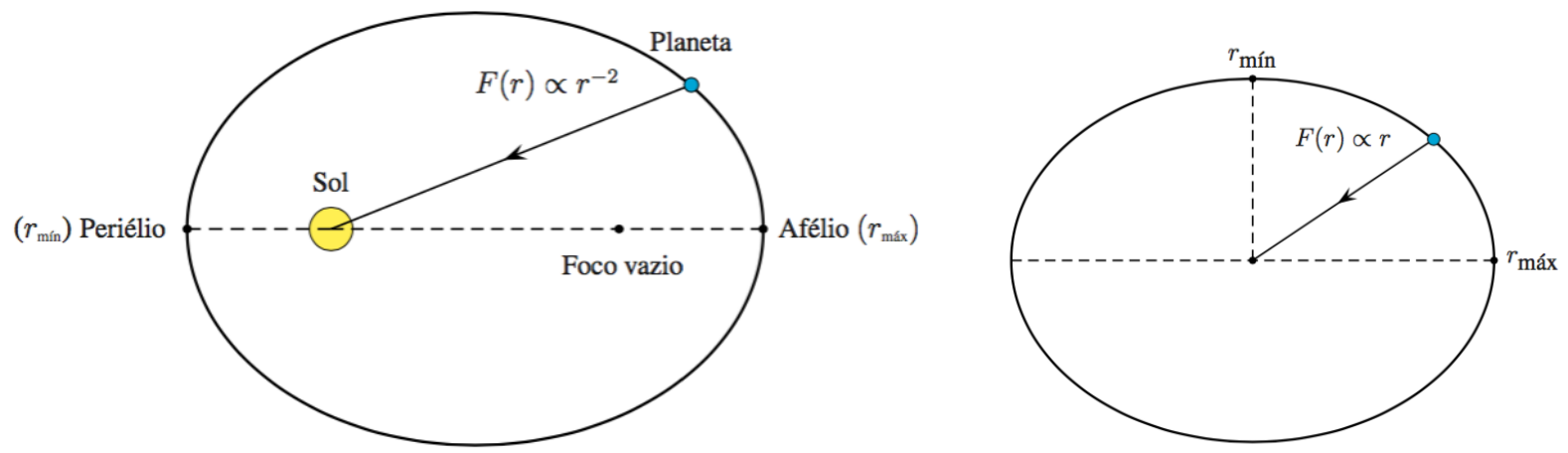

Figura 1: Para a lei da gravitação universal, $T_{\text {radial }} / T_{\text {orbital }}=$ Figura 2: Para uma lei de força do tipo do 1. Como a massa do Sol é muito maior do que a massa da Terra, o Sol é o centro de força e ocupa um dos focos da elipse. A excentricidade da órbita oscilador harmônico isotrópico: $T_{\text {radial }} / T_{\text {orbital }}=\frac{1}{2}$. elíptica da Terra é mostrada de modo exagerado.

4. A precessão dos equinócios faz com que o eixo de rotação da Terra descreva um cone no espaço com período igual a aproximadamente 26000 anos e se deve aos torques exercidos principalmente pelo Sol e pela Lua.

A precessão do periélio de um planeta acontece quando à lei da gravitação universal devemos acrescentar termos adicionais para levar em conta efeitos perturbativos observados pelos astrônomos. Por exemplo, o fato do Sol ser levemente oblato, leva a termos adicionais que quebram o caráter central da força que atua sobre um planeta. Outro exemplo é o efeito da gravitação relativística que faz com que tenhamos de adicionar à força $\propto r^{-2}$ que atua sobre um planeta, um termo $\propto r^{-4}$. A força continua central, mas agora não temos mais a garantia de que órbita será fechada.

Há vários métodos analíticos para descrever a precessão do periélio de um planeta, mas todos eles começam e vão muito além do que seria razoável discutir no ensino médio. No entanto, com os softwares disponíveis nos dias de hoje é possível curto-circuitar essas dificuldades e introduzir o tema e analisálo com os métodos numéricos subjacentes a estes softwares, como por exemplo o Modellus [7] que utilizaremos aqui para estudar o avanço do periélio de Mercúrio.

\subsection{A contribuição relativística à precessão de Mercúrio}

A história da precessão de Mercúrio está intimamente ligada ao nome do astronônomo francês Urbain Jean Joseph Le Verrier (1811 - 1877), o descobridor de Netuno [2].

Fervoroso defensor da teoria newtoniana da gravitação, Le Verrier, a partir de 1839 envolveu-se no cálculo da estabilidade do Sistema Solar e das órbitas planetárias. Por volta de 1841, sua atenção estava voltada para o problema da determinação precisa da órbita de Mercúrio. Este planeta é o mais próximo do Sol e sua órbita tem excentricidade razoável e um período curto.

Em 1859, Le Verrier concluiu que a órbita de Mercúrio precessa lentamente, o que era perfeitamente 

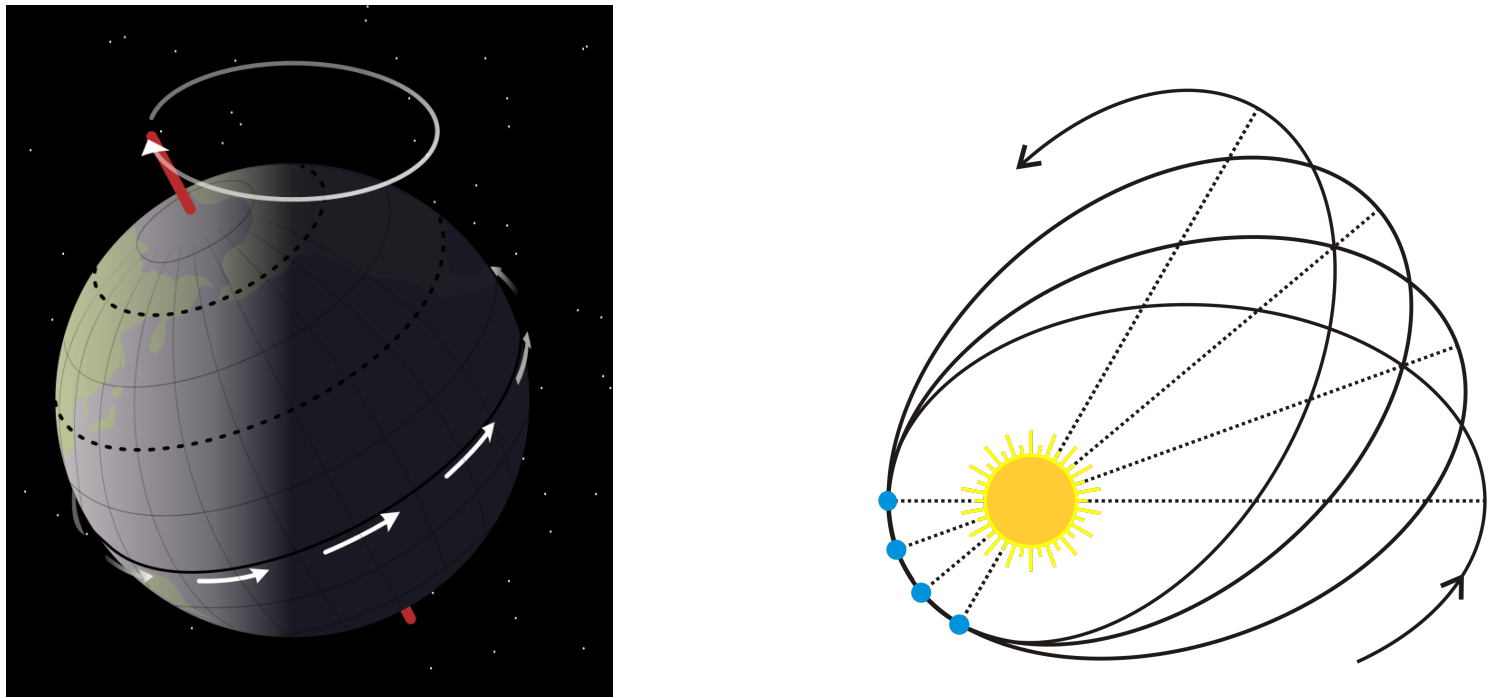

Figura 3: Precessão dos equinácios: como um pião, o Figura 4: Precessão apsidal de um planeta em relação eixo de rotação da Terra descreve um cone no espaço. (Robert Simmon, NASA GSFC (Earth ao Sol. (Robert Simmon, NASA GSFC (Earth Observatory /NASA) [Wikimedia Commons DoObservatory /NASA) [Wikimedia Commons Domínio Público]) mínio Público])

natural se a influência dos demais planetas fosse levada em conta. As observações feitas com os instrumentos da época revelavam que a precessão de Mercúrio era de 565 segundos de arco por século. Le Verriere obteve um valor de 527 segundos de arco, não conseguindo explicar a diferença de 38 segundos de arco entre os valores observados e calculados $(565-527)$.

\begin{tabular}{cc} 
Segs de arco por século & Origem \\
\hline \hline $531,63 \pm 0,69$ & Pert. grav. planetas \\
0,0254 & Oblaticidade do Sol \\
$42,98 \pm 0,04$ & Relatividade Geral \\
$574,64 \pm 0,69$ & Total teórico \\
$574,10 \pm 0,65$ & Observado \\
\hline \hline
\end{tabular}

Figura 5: Principais contribuições à precessão da órbita de Mercúrio. Os valores teóricos e experimentais são em referência ao ICRF - International Celestial Reference Frame - cuja origem localiza-se no baricentro do Sistema Solar.

Melhorias nas lentes e refinamentos matemáticos fizeram com que a diferença entre o que era observado e o que era calculado fosse de 38 para 43 segundos de arco. As tentativas de explicar esta discrepância levaram à hipótese da existência de um planeta entre o Sol e Mercúrio, Vulcano, que terminou sendo abandonada. Outra explicação proposta era atribuir uma massa $10 \%$ maior para Vênus. Isto poderia 
resolver o problema de Mercúrio, mas pertubaria a órbita da Terra, fato que não era observado. A verdade é que a lei da gravitação universal de Newton não era capaz de revelar o que estava por trás desse valor, que só foi explicado em 1916 por Einstein tornando-se em um dos primeiros testes observacionais da versão relativıśtica da gravitação.

Esses 43 segundos de arco correspondem à contribuição relativística à precessão de Mercúrio. O que isso significa? Significa que o Sol, por ser uma estrela massiva, deforma o espaço-tempo ao seu redor e essa deformação contribui para a precessão dos planetas, especialmente para a de Mercúrio, pela sua proximidade com Sol.

Adicionando a contribuição relativística à lei da gravitação universal de Newton, teremos:

$$
F(r)=-\left(\frac{G M m}{r^{2}}+\frac{3 G M L^{2}}{m r^{4} c^{2}}\right),
$$

onde $G$ é a constante da gravitação universal, $M$ é a massa do Sol, $m$ é a massa do planeta, $L$ é o módulo do momento angular em relação ao Sol, $r$ é a distância entre o Sol e o planeta e $c$ é o módulo da velocidade da luz no vácuo.

Como estamos trabalhando apenas com forças centrais e estas são conservativas, não haverá torques ou dissipação de energia: o momento angular e a energia mecânica do sistema serão então constantes de movimento. Assim, o fator $3 G M L^{2} /\left(m c^{2}\right)$ é constante. Convém rescrever a força atuante no planeta na forma:

$$
F(r)=-\frac{k}{r^{2}}-\frac{\gamma}{r^{4}}
$$

onde $k=G M m$ e $\gamma=3 \frac{G M L^{2}}{m c^{2}}$. Como a magnitude dessa perturbação é muito pequena, $\frac{k}{r^{2}}>>\frac{\gamma}{r^{4}}$.

A partir deste ponto podemos levar nossos alunos ao laboratório de informática e trabalhar não só a $1^{\mathrm{a}}$ lei de Kepler, mas também explorar, de maneira qualitativa, o fenômeno da precessão.

\section{Ilustrando a $1^{\text {a }}$ lei de Kepler e introduzindo a precessão: uma sugestão de roteiro}

No que vem a seguir, sugeriremos alguns passos sequenciais para a utilização do Modellus no ensino de gravitação. O roteiro está dividido em duas etapas: a primeira etapa consiste no uso do programa para mostrar como se comporta um planeta sob inflûencia apenas do Sol e poderá ter suas próprias conclusões, ao invés de o professor simplesmente enunciar a $1^{\text {a }}$ lei de Kepler. A segunda etapa diz respeito à precessão e deve acontecer sequencialmente [8].

\subsection{Etapa 1 - Ilustrando a $1^{\mathrm{a}}$ lei de Kepler}

É uma prática geral em sala de aula introduzir as leis de Kepler enunciando-as formalmente, logo a atividade prática inicial deve consistir em mostrar que a lei da gravitação universal de Newton leva às órbitas elípticas. A seguir sugerimos um roteiro para o professor. 
(a) Inicie a atividade representando um plano cartesiano com o Sol na origem e um planeta em um ponto arbitrário do plano $x y$. Represente a força gravitacional $\mathbf{F}$, assim como suas componentes cartesianas $F_{x}$ e $F_{y}$, (veja a Figura 6).

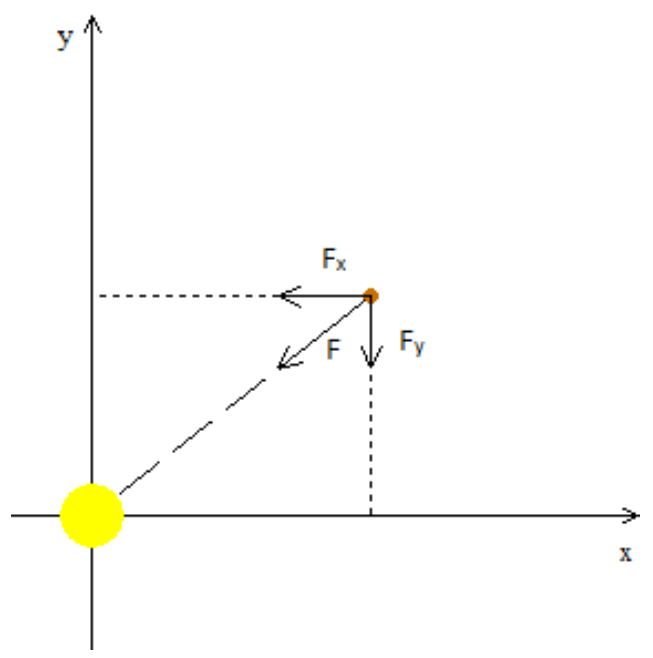

Figura 6: Como a massa do Sol é muito maior do que a massa do planeta, o centro de forçado sistema Sol-planeta localiza-se no Sol, que por sua vez está fixo na origem do sistema de coordenadas cartesiano.

(b) Próximo passo é desenvolver com os alunos a expressão das componentes cartesianas da força:

$$
F_{x}=-F \frac{x}{\left(x^{2}+y^{2}\right)^{1 / 2}}
$$

$\mathrm{e}$

$$
F_{y}=-F \frac{y}{\left(x^{2}+y^{2}\right)^{1 / 2}} .
$$

Fazendo uso da lei da gravitação universal e da $2^{\mathrm{a}}$ lei de Newton, podemos obter as equações de movimento:

$$
a_{x}=-G M \frac{x}{\left(x^{2}+y^{2}\right)^{3 / 2}}
$$

$\mathrm{e}$

$$
a_{y}=-G M \frac{y}{\left(x^{2}+y^{2}\right)^{3 / 2}}
$$

onde $a_{x}$ e $a_{y}$ são as componentes da aceleração a que o planeta está submetido.

(c) Sabendo as expressões das componentes cartesianas da aceleração podemos implementá-las no Modellus. Ao abri-lo aparecerá uma aba onde se lê: Modelo Matemático. É nesse espaço que devemos colocar as equações que regem o nosso sistema, conforme mostra a Figura 7. 


\begin{tabular}{|l||}
\hline Modelo Matemático \\
\hline$\frac{\mathrm{d} x}{\mathrm{~d} t}=v x$ \\
$\frac{\mathrm{d} y}{\mathrm{~d} t}=v y$ \\
$\frac{\mathrm{d} v x}{\mathrm{~d} t}=-x \times\left(x^{2}+y^{2}\right)^{(-1.5)}$ \\
$\frac{\mathrm{d} v y}{\mathrm{~d} t}=-y \times\left(x^{2}+y^{2}\right)^{(-1.5)}$ \\
\hline Parâmetros Condições iniciais \\
\hline
\end{tabular}

Figura 7: Modelo matemático. Como estamos preocupados com a parte qualitativa do problema e por limitações do programa, podemos escolher GM numericamente igual a 1.

Após escrever as equações conforme mostra a Figura 7, peça aos alunos para que cliquem no item interpretar.

(d) Para finalizar essa primeira atividade, basta escolher as condições iniciais e clicar em play:

\begin{tabular}{|c|c|c|c|c|}
\hline Parâmetros & \multicolumn{3}{|c|}{ Condições Iniciais } & \\
\hline & \multirow{3}{*}{$\begin{array}{l}x= \\
v x=\end{array}$} & $\square$ Caso1 & $\square$ Caso2 & $\square$ Caso3 \\
\hline Iguais & & 1.00 & 0.00 & 0.00 \\
\hline Iguais & & 0.00 & 0.00 & 0.00 \\
\hline Iguais & \multirow{2}{*}{$\begin{array}{l}y= \\
y=\end{array}$} & 0.00 & 0.00 & 0.00 \\
\hline Iguais & & 1.10 & 0.00 & 0.00 \\
\hline
\end{tabular}

Figura 8: Exemplo de condições iniciais. Outras escolhas de condições iniciais também garantem uma órbita elíptica, desde que a energia mecânica seja negativa, assegurando estados ligados.

A Figura 9 mostra a trajetória gerada pelo programa: 


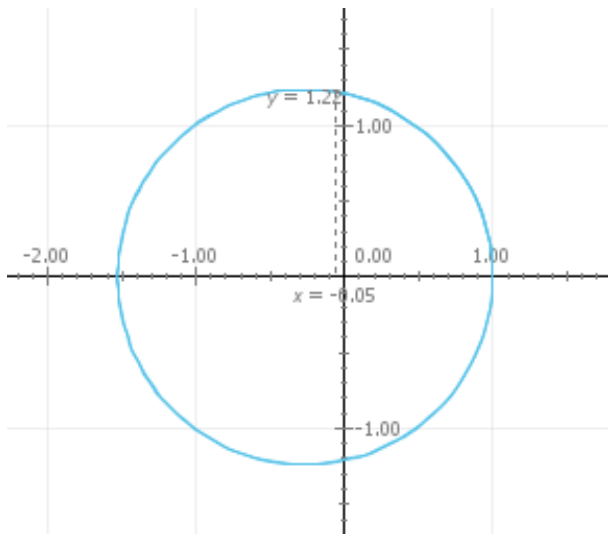

Figura 9: Órbita de uma partícula sujeita exclusivamente a uma força atrativa proporcional a $1 / r^{2}$ em determinados níveis de energia. Note que o centro de força não coincide com centro geométrico da elipse.

A partir desse resultado os alunos poderão compreender com maior facilidade a $1^{\mathrm{a}}$ lei de Kepler.

\subsection{Etapa 2 - Introduzindo a precessão}

Uma vez comprovada a $1^{\text {a }}$ lei de Kepler, é o momento de falar um pouco sobre perturbações e seus efeitos sobre a órbita de um planeta. Em particular, deve-se falar sobre o problema da precessão do periélio de Mercúrio e os famosos 43". Deve-se enfatizar que a solução de Einstein descarta completamente outras soluções newtonianas como, por exemplo, a existência de um planeta entre o Sol e Mercúrio, (Vulcano) ou um aumento de $10 \%$ da massa de Vênus que poderia explicar a precessão de Mercúrio, mas afetaria a órbita da Terra, algo que não era observado.

Eis então aqui a nossa sugestão para um roteiro de introdução à precessão de Mercúrio.

(a) O professor pode dar início à aula formulando a pergunta: "E se houver uma força adicional atuando no planeta?", explicando que o sistema Sol-Mercúrio na verdade não está isolado, em razão da influência dos outros planetas e do efeito da deformação do espaço - tempo (efeito relativístico), que gera perturbações na órbita. Como Mercúrio está mais próximo do Sol, é o que sofre mais com esse efeito.

A seguir, o professor deve "convencer" os alunos que, com a contribuição relativística, a força efetiva que atua sobre Mercúrio passa a ser:

$$
F(r)=-\left(\frac{G M m}{r^{2}}+\frac{\gamma}{r^{4}}\right)
$$

onde $\gamma$ é uma constante positiva. Deve explicar também que o termo perturbativo é muito pequeno, ou seja, $\frac{G M m}{r^{2}}>>\frac{\gamma}{r^{4}}$. Seguindo o mesmo raciocínio da atividade anterior, os alunos devem escrever as componentes da aceleração que atua sobre Mercúrio em um ponto $P(x, y)$ de sua órbita: 


$$
a_{x}=-G M \frac{x}{\left(x^{2}+y^{2}\right)^{3 / 2}}-\gamma \frac{x}{\left(x^{2}+y^{2}\right)^{5 / 2}}
$$

$$
a_{y}=-G M \frac{y}{\left(x^{2}+y^{2}\right)^{3 / 2}}-\gamma \frac{y}{\left(x^{2}+y^{2}\right)^{5 / 2}} .
$$

(b) Uma vez inseridas as equações no Modellus, basta clicar no botão interpretar e digitar as mesmas condições inicias da primeira atividade, (ver Figura 8). Por último, clique em Run (rodar). No Modellus, as equações que representam a trajetória de Mercúrio são mostradas na Figura 10.

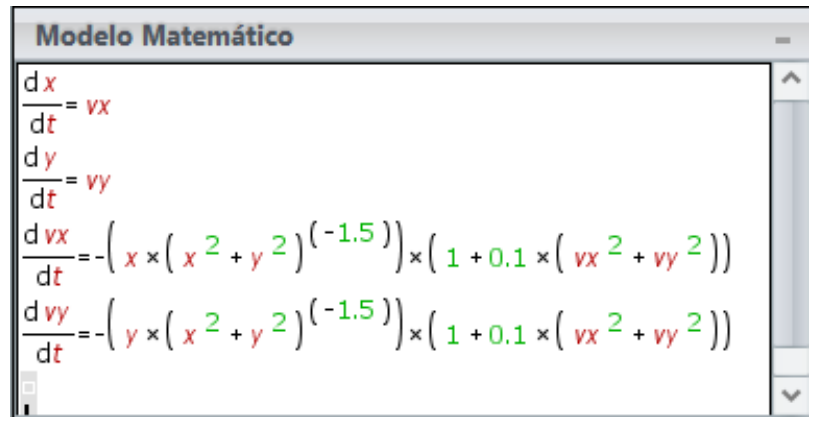

Figura 10: Como estamos preocupados com a parte qualitativa - o aluno observar que a órbita não fecha -o professor pode explicar aos aluno que, ao colocar as equações no programa, a força perturbativa deve ter módulo bem menor que a gravitacional e, para garantir que isso aconteça, multiplicou o termo perturbativo por 0.1. Note que a escolha de $\gamma$ depende das condições iniciais, pois depende do momento angular.

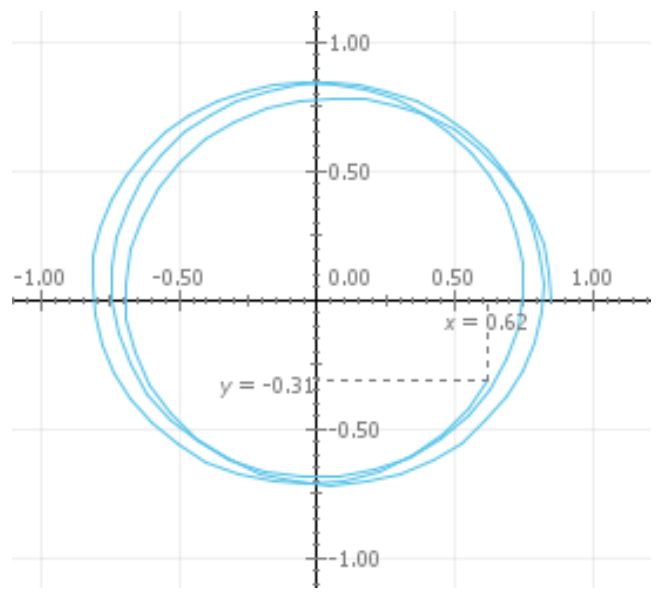

Figura 11: Órbita com precessão.

(c) Agora peça aos alunos que examinem a órbita calculada pelo Modellus. O objetivo é que estes percebam que a órbita já não fecha mais, embora ainda seja limitada. Após essa conclusão, explique que a perturbação, isto é: o termo adicional, é o responsável pela precessão (axial) da órbita. 
(d) Como última tarefa peça aos alunos que usem o valor da precessão de Mercúrio 43"/século para mostrar que a linha das apsides, isto é: o eixo maior da órbita elíptica de Mercúrio demora 3 milhões de anos para dar uma volta completa [9].

Nossa estimativa é que a aula tenha uma duração aproximada de uma hora e meia, mas nada impede que professor divida o tema em duas aulas, uma trabalhando em cima da força gravitacional newtoniana afim de obter a $1^{\text {a }}$ lei de Kepler e outra focando no fenômeno da precessão.

\section{Observações finais}

Um dos autores (RFFC) do presente trabalho testou o roteiro sugerido com três turmas de 25 alunos cada, em uma escola da rede federal na cidade de Santo Antônio de Pádua, estado do Rio de Janeiro, em novembro de 2016 com resultados satisfatórios no que diz respeito ao engajamento dos alunos; um maior entendimento da gravitação também foi observado. O importante é que ao utilizar o Modellus, o professor pode trabalhar a $1^{\mathrm{a}}$ lei de Kepler e o fenômeno da precessão com seus alunos de uma maneira não tradicional dando-lhes a oportunidade de deixar a passividade de lado, tornando-os mais participativos na sala de aula.

Como desafio, o professor pode sugerir aos seus alunos que estudem o oscilador harmônico isotrópico perturbado for uma força $\propto-r^{-3}$, ver a equação (2) em [10]:

$$
F(r)=-\left(k r+\frac{\gamma}{r^{3}}\right)
$$

onde $k$ e $\gamma$ são constantes. Os alunos devem escolher o valor das constantes, as condições iniciais seguindo os passos descritos para o caso do problema de Kepler perturbado. O professor deve lembrar aos alunos que como antes, a escolha judiciosa das constantes é uma concessão às limitações do software e permite visualizar claramente a órbita perturbada.

Esperamos que os argumentos apresentados aqui sejam convincentes, estimulem nossos colegas professores de física e sejam úteis no processo de aprendizagem dos nosso alunos.

\section{Agradecimentos}

Os autores agradecem ao Dr. V. Soares pela leitura atenta do manuscrito original e sugestões pertinentes.

\section{Referências}

[1] D’Inverno, R.: Introducing Einstein's Relativity Clarendon Press Oxford 1992.

[2] Baum, R. \& Sheehan, W.: In Search of Planet Vulcan: The Ghost in Newton's Clockwork Universe. Plenum, NY 1997. 
[3] Goldstein, H.; Poole, C. \& Safko, J.: Classical Mechanics 3 ${ }^{\text {a }}$ ed. Addison-Wesley, New York 2002.

[4] Gregory, D. R.: Classical Mechanics Cambridge University Press, Cambridge 2006.

[5] Chin, S. A.: A truly elementary proof of Bertrand's theorem Am. J. Phys. 83 (4) 320-323 2014.

[6] Santos, F. C.; Soares, V. \& Tort A. C.: Uma tradução da demonstração original do teorema de Bertrand. Revista Brasileira de História da Ciência. 4 n.1 92-97 (2011).

[7] http://www.if.ufrgs.br/computador-ensino-fisica/modellus/modellus-Introducao.htm Acessado em 02/08/2017.

[8] Cunha, R. A Gravitação e a Precessão de Mercúrio: um texto para professores do Ensino Médio. Rio de Janeiro. 133p. Dissertação de mestrado, Programa de Pós-Graduação em Ensino de Física, Instituto de Física, Universidade Federal do Rio de Janeiro, Rio de Janeiro. 2017.

[9] Asimov. I.: The planet that wasn't, disponível em http://geobeck.tripod.com/frontier/planet.htm. Acessado em 06/09/2017.

[10] Tort A.; Farina, C. \& Medeiros, O.: Perturbed Isotropic Harmonic Oscilator, Eur. J. Phys 10 220-223 1989. 